\title{
Atelectasia pulmonar en el lactante
}

\author{
Dres.: Jaime Valenzuela B.," Sylvia Medina P., ** Humberto Broitman D.***.
}

\begin{abstract}
Atelectasis is defined as collapse of aerated lung tissue, first described in 1819 and reproduced experimentally by Trambe in 1845 .

Ten infants with this pathology are analized, over a three year period (1976-1979), in the Pediatric In-Patient Unit, Hospital Guillenno Grant Benavente, Concepción.

Six of them are under twelve months of age and in eight ehildren atelectasia followed bronchopneunonia. Three cases evolved favorably in two weeks, five cases resolved in four weeks and two cases persisted after three months of evolution. All of them received kinesic treatment, with improvement in eight.
\end{abstract}

La Atelectasia Pulmonar es el colapso del tejido aireado del pulmón. Fue descrito por primera vez en 1819 por Laennec y reproducido experimentalmente por Trambe en 1845.

La incidencia de las atelectasias adquiridas en la niñez depende de: la edad del paciente, el tipo de población tomada como muestra, el estado de alerta de los médicos para descubrirlas y la utilización de diferentes técnicas radiológicas.

James y col., analizaron las atelectasias radiologicas desde los años 1945 a 1952, encontrando que la coqueluche fue la etiología de mayor incidencia, con un $18,9 \%$ y diversas infecciones del tracto respiratorio con un $58,2 \%$.

Esta situación ha cambiado en los últimos años con el mejor manejo profiláctico y terapéutico de las enfermedades infecto-contagiosas; destacándose actualmente como etiologias prevalentes a las infecciones parenquimatosas pulmonares y al síndrome bronquial obstructivo crónico; afectando la primera condición a los menores de dos años y la segunda, a los mayores.

Asimismo, cabe mencionar como causa frecuente de atelectasia al vómito y la aspiración de cuerpo extraño en los menores de dos años.

En los niños menores de dos años, la atelectasia puede desarrollarse e instalarse en horas, no asi en el niño mayor en que el proceso es más lento. debido a que la luz bronquial es mayor, la estructura cartilaginosa más firme y las paredes toracicas más rígidas.

\footnotetext{
"Servicio Pediatría Hospital Gmo. Grant Benavente Concepción. Departamento Pediatria. Esc. de Medicina Universidad de Concepción.

* Servicio Pediatría Hospital Gmo. Grent Benavente Concepción. ***Becado Pediatría, Hospital Gmo. Grant Benavente Concepción.
}

La Atelectasia Pulmonar se produce por la obstrucción completa bronquial, la que debe persistir el tiempo necesario para permitir la reabsorción del aire del tejido colapsado. Esta reabsorción depende del flujo sanguíneo pulmonar y de la solubilidad de los gases. El aire atmosférico, N2 y helio lo bacen en dos o tres horas; en cambio, el oxígeno se absorbe en unos pocos minutos.

Después del colapso la ventilación del parénquima afectado se hace mínima, mientras que la perfusión sanguínea puede estar disminuida sólo discretamente, determinando shunt arteriovenoso. Además de esta complicación hipóxica (shunt) la infección del tejido atelectasiado es frecuente, debido a la acumulación de secreciones, así como también a la alteración del surfactante pulmonar. Las zonas vecinas a la atelectasia se distienden, por lo que pequeñas atelectasias pueden ser no evidenciables radiológica ni clínicamente. En las grandes atelectasias el corazón y el mediastino pueden estar desplazados y el diafragma puede ascender hacia el sector colapsado.

La sintornatología clínica varia según sea la causa del colapso pulmonar. En general las que se producen en el curso de una bronconeumonía, bronquitis obstructiva, bronquiolitis, tuberculosis, linfomas, neoplasias. no producen cambios en el cuadro clínico de fondo, a menos que se obstruya uno de los bronquios principales, con lo cual aumenta la sintomatología respiratoria, o se inicia, 1,2

Los signos radiológicos sugerentes de atelectasia son:

a) La cisura se distiende más allá de los límites normales y la convexidad cambia a concavidad.

b) Reducción del segmento o lobulo afectado.

c) Desplazamiento del hilio pulmonar hacia el lado afectado. 
Las atelectasias pueden afectar cualquier lóbulo o segmento pulmonar. En los niños pequeños es el lóbulo superior derecho el más afectado, debido probablemente a una mayor incidencia de

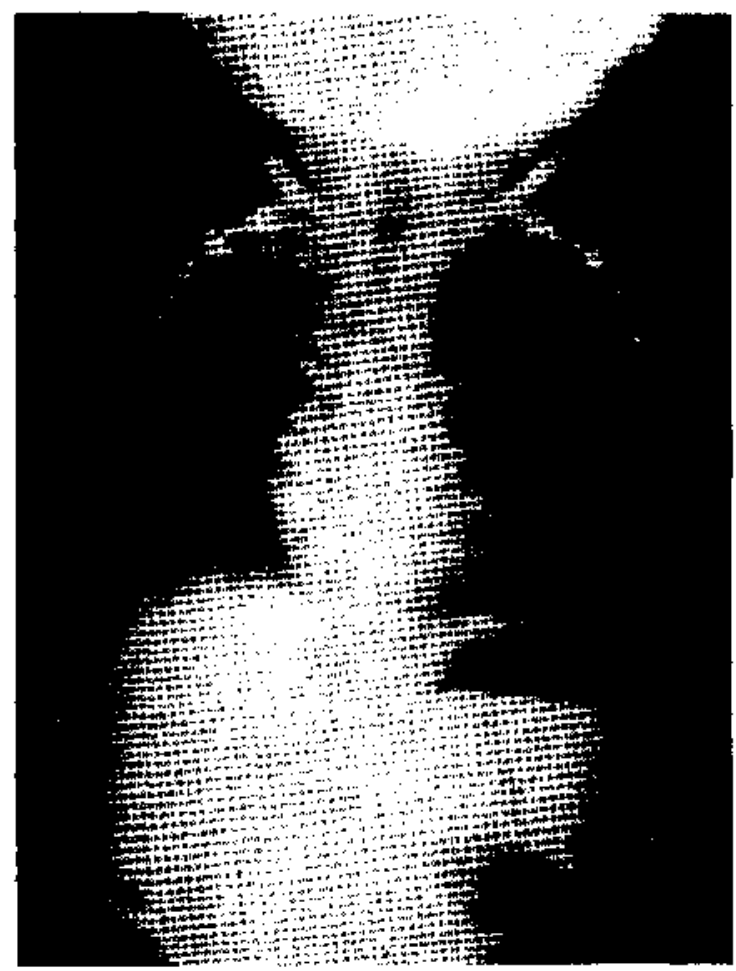

Foto 1

\section{MATERIAL Y METODO}

Se revisan las fichas clinicas de 10 lactantes, que presentaron atelectasia pulmonar, en un período de tres años, junio 1976 a julio 1979 , en la Unidad de Lactantes del Servicio de Pediatría Hospital Guillermo Grant Benavente. Se efectúa un análisis clínico de esta patologia y uma revisión del tema, que ha sido escasamente informado en nuestro medio.

Las fichas clinicas correspondieron a cinco varones y cinco mujeres.

\section{RESULTADOS}

En el gráfico 1 se analiza la edad de nuestros niños. El $30 \%$ eran menores de seis meses y el $90 \%$ menores de 18 meses.

En el gráfico 2 se analizan las causas de atelectasias. En 8 casos ( $80 \%$ ) fue secundaria a bronconeumonia, en uno a síndrome bronquial obstructivo y el restante a malformación bronquial. malformaciones bronquiales a ese nivel. Esto constituye el llamado Sindrome del Lobulo superior derecho. ${ }^{4}$ (Fotos 1 y 2 ).

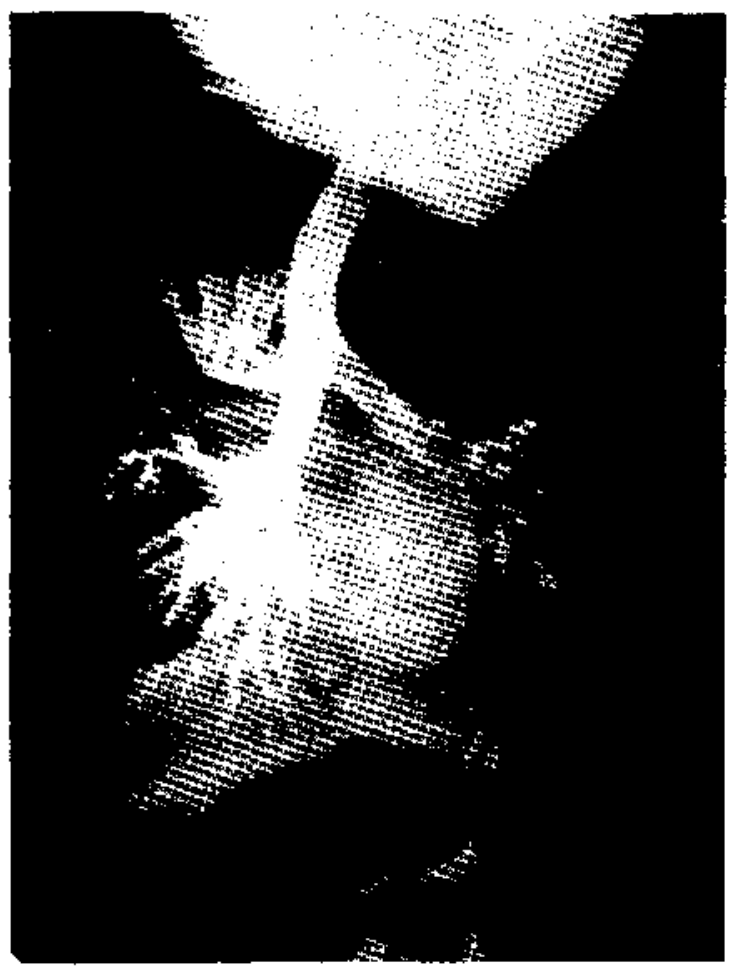

Foto 2

\section{Gráfico 1}

ATELECTASIA PULMONAR EN EL LACTANTE EDAD Y SEXO

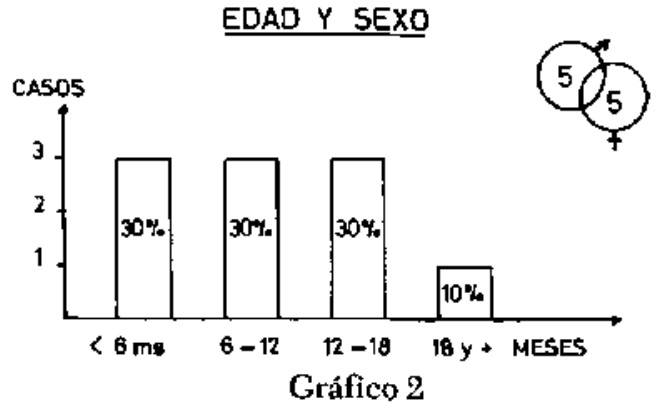

\section{ATELECTASIA PULMONAR EN EL LACTANTE EI IOPATOGENIA}

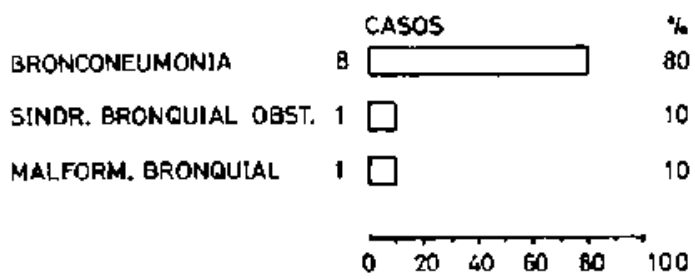


En el gráfico 3 se estudia la localización radiológica del colapso pulmonar. Este correspondióal l6bulo superior derecho en 7 casos (70\%) y los tres restantes (30\%) al lóbulo inferior izquierdo.

En el gráfico 4 se observan los días de evolución previa de enfermedad. Tres de ellos (30\%) tuvieron evolución menor a 7 djas. Uno presentó quince días de evolución, $y$ en cinco la evolución (50\%) fue mayor de 28 días. En el restante se ignora.

\section{Gráfico 3}

Atelectasia pulmonar en el lactante localización

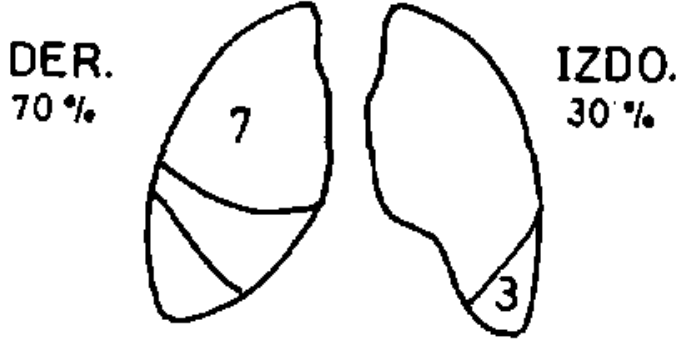

Gráfico 4

\section{ATELECTASIA PULMONAR EN EL LACTANTE DIAS EVOLUCION PREVIA}

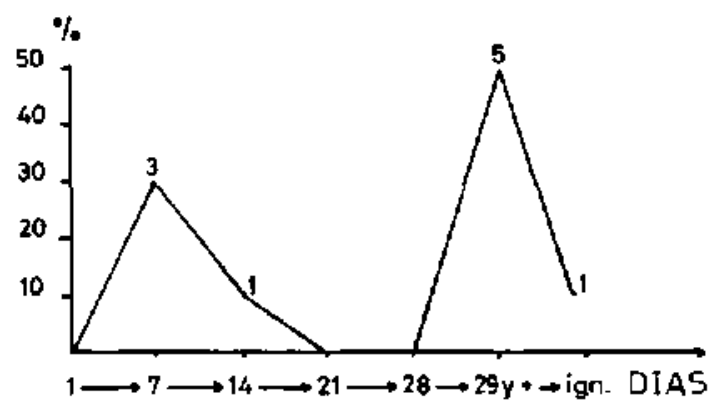

En gráfico 5 se analiza el tratamiento efectuado a los 10 lactantes con atelectasia pulmonar.

Se destaca el uso de kinesioterapia en $100 \%$ de ellos y el empleo de antibióticos en el $90 \%$. La broncoaspiración realizada por otorrinolaringólogo, con laringoscopio y sonda nelaton, se efectuó en un $60 \%$ de los casos y en dos casos la respuesta fue favorable.

En el gráfico 6 se analizan los antecedentes patológicos previos de los 10 lactantes. Se destaca que 4 casos $(40 \%)$ no presentan antecedentes de importancia, ел cambio en 4 niños (40\%) existe el antecedente de bronquitis recidivante desde los primeros meses de vida.

\section{Gráfico 5}

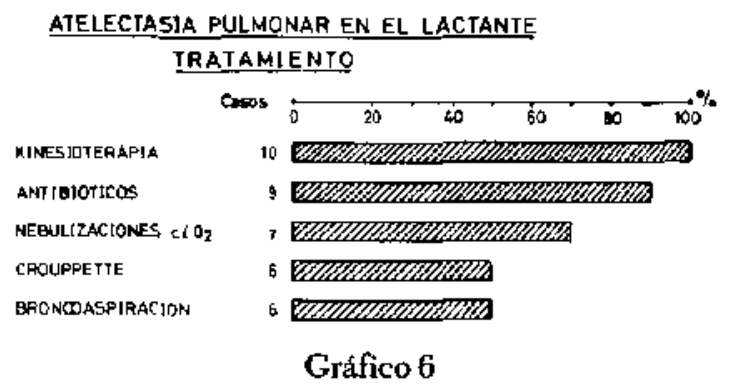

ATELECTASIA PULMONAR EN EL LACTANTE ANTECEDENTES PREVIOS CASOS

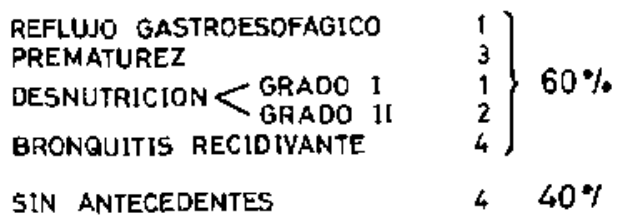

En el gráfico 7 se analizan los dfas de evolución de la atelectasia. Tres de los niños $(30 \%)$ tuvieron uma evolución rápida, inferior a catorce días; en cambio, cinco de ellos (50\%) presentaron ила evolución prolongada de un mes o más. Dos casos (20\%) tuvieron un curso crónico con más de tres meses de evolución.

\section{Gráfico 7}

Atelectasia pulmonar en el lactante días de evolución de la Atelectasia

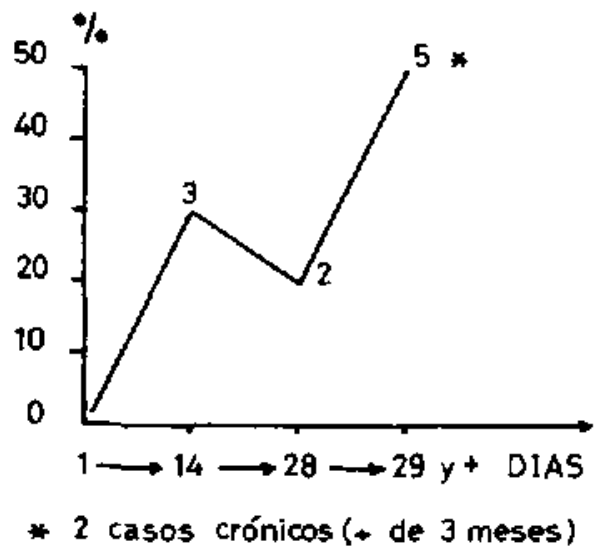




\section{COMENTARIO}

La Atelectasia Pulmonar es una patología de frecuente presentación en el curso de las bronconeumonías del lactante menor, la cual agrava y prolonga el curso evolutivo de ellas, tal como se aprecia en ocho de nuestro niños.

En su manejo es fundamental la sospecha clínjca, su confirmación radiológica para ir a una pronta broncoaspiración, realizada con una técnica adecuada. Esto explicaría el bajo rendimiento obtenido en nuestro material, ya que sólo en dos lactantes mayores se logró un resultado satisfactorio con dicha técnica. En los restantes, la respuesta no fue la esperada debido quizás a la ubjcación de la atelectasia y la técnica empleada. Por ello se recomienda actualmente el uso de catéteres angiográficos radio-opaco, introducidos bajo pantalla fluoroscópica. ${ }^{3}$ (Foto 3 y 4).

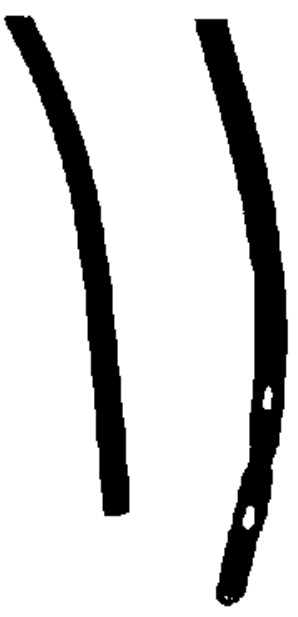

Foto 3

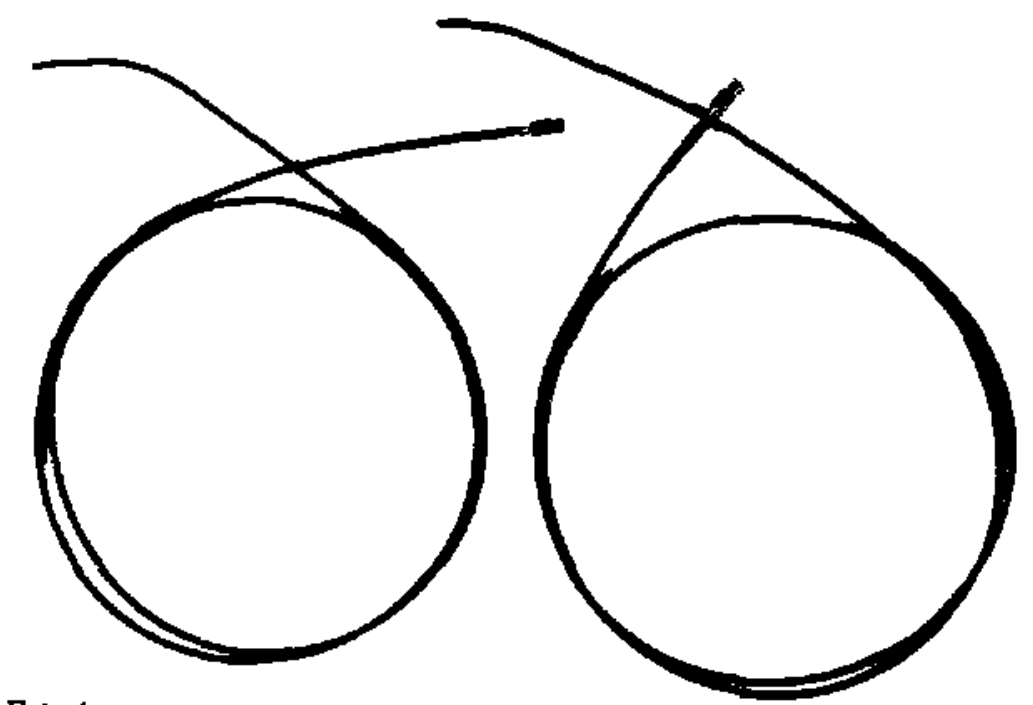

Foto 4
En los riños portadores de Síndrome bronquial recurrente, que presentan atelectasia del lóbulo superior derecho, se recomíenda la broncografía, ya que frecuentemente se asocian a malformaciones bronquiales, tal como se observó en uno de nuestros niños.

En el manejo de toda Bronconeumonía o Bronquitis obstructiva del lactante, sobre todo menor de un año, es fundamental el manejo correcto médico-kinésico, para evitar el taponamiento bronquial por secreciones, causal de atelectasia en la inmensa mayoría de los niños; y no realizarla exclusivamente cuando esta complicación ya está presente.

Dada la escasa muestra analizada, no se pueden entregar porcentajes estadisticamente significativos, sino tan sólo sugerencias etiológicas, conducta terapéutica y control evolutivo de estos niños.

Lo más destacado, creemos, sin embargo, es la permanente sospecha clínica y la broncoaspiración precoz (sin anestesia) dirigida radiológicamente.

\section{RESUMEN}

La Atelectasia Pulmonar es el colapso del tejido aireado del pulmón. Descrito por primera vez por Laennec en 1819, como hallazgo de autopsia y reproducido experimentalmente por Trambe en 1845.

Se estudian 10 casos clínicos en lactantes que presentaron Atelectasia Pulmonar en un período de tres años, junio 1976 a junio 1979, en la Unidad de Lactantes, Servicio de Pediatria, Hospital Guillermo Grant Benavente.

Se efectúa un análisis clínico de esta patología y una revisión del tema, que ha sido escasamente estudiado en el lactante.

Seis de los lactantes son menores de 1 año; en ocho de ellos la atelectasia fue secundaria a bronconeumonia. La evolución fue mayor de un mes en 3 casos, menos de l mes de evolución en cinco.

En todos se realizó tratamiento médico kinésico, logrando mejoría en 8 . En los dos restantes la patología en referencia siguió un curso crónico, 
uno de los cuales presenta malformación bronquial.

Mortalidad no se registró en este grupo.

\section{REFEHENCLAS}

ICherniack, R.M.; Louis Chemiak; Amold Naimark, "Respiración normal y patologica”. Ed. Toray $2^{2}$. Ed. Barcelona, 1975.

${ }^{2}$ Kendig Eduin. "Enfenmedades del Aparato Respiratorio en la Infancia". Ed. La Médica lema Ed. Argentina, 1971.

${ }^{3}$ Gatris, A.G.; White, John I.: OH Koak Sang. "A Bedside was- houttechnique for atelectasis in infants". Am. J. Dischild L27: 324, 1974 .

${ }^{4}$ Kollar D. "Die therapeutishe bedeu tung der Bronchussaugdrainage im Sāuglingsalter". Monatsschr Kinder heilkd 124: $71,1976$.

${ }^{5}$ Schwartz, ML et al. "The effect of Steroids on adaptation to atelectasis shunting". J. Thorac Cardiovasc Surg 68: 822, 1974.

FErlich Robert; Amon Rica, "The intemitient endotracheal intubation technique for the traitment of recurrent atelectasis". Pediatrics 50: 144, 1972.

7 Wanner Adam, et al. "Bedside bronco fiber scopy for atelectasis and lung abscess". Jama 224: 1281, 1973. 